Man and Nature

L'homme et la nature

\title{
Le supplice de Servet : Voltaire historien et les droits de la personne
}

\author{
James Forsythe MacLean
}

Volume 10, 1991

URI : https://id.erudit.org/iderudit/1012628ar

DOI : https://doi.org/10.7202/1012628ar

Aller au sommaire du numéro

Éditeur(s)

Canadian Society for Eighteenth-Century Studies / Société canadienne d'étude du dix-huitième siècle

ISSN

0824-3298 (imprimé)

1927-8810 (numérique)

Découvrir la revue

Citer cet article

MacLean, J. F. (1991). Le supplice de Servet : Voltaire historien et les droits de la personne. Man and Nature / L'homme et la nature, 10, 113-120.

https://doi.org/10.7202/1012628ar

Copyright (C) Canadian Society for Eighteenth-Century Studies / Sociéte canadienne d'étude du dix-huitième siècle, 1991
Ce document est protégé par la loi sur le droit d'auteur. L'utilisation des services d'Érudit (y compris la reproduction) est assujettie à sa politique d'utilisation que vous pouvez consulter en ligne.

https://apropos.erudit.org/fr/usagers/politique-dutilisation/ 


\section{Le supplice de Servet: Voltaire historien et les droits de la personne}

D'après ce qu'il raconte dans l'article 'Dogmes' de son Dictionnaire philosophique, le 18 février de l'an 1763 Voltaire fut transporté au ciel. Il y eut l'honneur d'assister au jugement de Jean Calvin. Au cours de ce procès céleste, le réformateur genevois se vantait de ses apports à l'amélioration du monde: il avait, par exemple, condamné la peinture et la sculpture, et il avait démontré le caractère diabolique du menuet. Alors qu'il donnait ces explications, 'on vit auprès de lui, écrit Voltaire un bûcher enflammé; un spectre épouvantable, portant au cou une fraise espagnole à moitié brûlée, sortait du milieu des flammes avec des cris affreux. "Monstre, s'écriait-il, monstre exécrable, tremble! reconnais ce Servet que tu as fait périr par le plus cruel des supplices, parce qu'il avait disputé contre toi sur la manière dont trois personnes peuvent faire une seule substance."'11 Dans cette scène macabre l'antitrinitaire Michel Servet, exécuté à Genève en 1553, symbolise tous les martyrs qui au cours des siècles ont été mis à mort en raison de leurs seules croyances religieuses. Dès la période où il rédigeait la Henriade (publiée en 1728), le massacre de la Saint-Barthélemy, en 1572, avait été dans l'esprit de Voltaire l'exemple par excellence de $l^{\prime}$ intolérance fanatique. ${ }^{2}$ On dit que chaque année au 24 août le philosophe souffrait d'un accès de fièvre. ${ }^{3}$ Cependant, à partir du moment où il s'installa dans les environs de Genève, le bûcher de Servet semble incarner de plus en plus pour Voltaire la quintessence du fanatisme. 'Il n'y eut point, écrit-il, dans votre st Bathélemi d'assassinat plus cruellement exécuté. ${ }^{4} E t$ revenant à son propre siècle le philosophe déclare que 'Le meurtre de Calas est une action très pardonnable en comparaison. ${ }^{5}$

Dans l'Essai sur les moeurs l'auteur consacre un chapitre entier à l'exécution de Servet. C'est un sujet qui apparaît assez souvent dans les écrits et la correspondance de Voltaire pendant les années 1750 et 1760. Ses opinions sur cette affaire, ainsi que la dispute au sujet du théâtre, provoquèrent une brouille entre Voltaire et les autorités genevoises. Au début de son séjour aux Délices, en 1755, Voltaire chantait les louanges de Genève et de ses libertés. Mais Voltaire se trouve bientôt à couteaux tirés avec le clergé et les milieux dirigeants de Genève en raison de ses 
attaques contre Calvin. Un Genevois influent, David Rival, lui communique un poème qui a le thème suivant:

Servet eut tort, et fut un sot

D'oser dans un siècle falot

S'avouer antitrinitaire.

Et notre illustre atrabilaire

Eût tort d'employer le fagot

Pour réfuter son adversaire. ${ }^{6}$

Voltaire montre que le cas de Servet le préoccupe jusque dans une lettre commerciale qu'il adressa en 1759 au marchand Jean Robert Tronchin. D'abord il se plaint: 'En fait de vins je suis le plus malheureux homme qui respire. Les 6 tonnaux de Languedoc sont arrivez absolument gâtez.' Ensuite il explique pourquoi il a besoin de son indépendance et de son domaine: 'je me ruine, je le sçais bien: mais ... il arrivera infailliblmnt [sic] que les prêtres de Baal dans trente ou quarante ans d'icy voudront me faire brûler comme Servet et Antoine, et je veux être en état de les faire pendre aux crénaux de mes châteaux. ${ }^{7}$

De tous les individus qui ont été mis à mort pour leurs croyances, pourquoi Voltaire attache-t-il tant d'importance à Servet? Pour répondre à cette question il faudra d'abord demander qui fut Michel Servet et pourquoi Jean Calvin le livra au bourreau.

Celui que les hallebardiers de Genève avaient escorté au bûcher en octobre 1553 était un des plus savants hommes de la Renaissance du XVIe siècle. Michel Servet de Villeneuve en Espagne est célèbre dans l'histoire de la médecine par sa découverte de la circulation pulmonaire du sang. Fait curieux, le malheureux n'était arrivé à Genève que la veille de son arrestation: seulement quatre mois avant il avait réussi à s'évader d'une prison appartenant à l'Inquisition romaine dans la ville française de Vienne, et il se dirigeait alors vers Naples, où il espérait exercer sa profession de médecin tout en gardant l'incognito, comme il l'avait fait pendant de longues années. ${ }^{8}$

Aussi invraisemblable que cela puisse paraître, c'était Jean Calvin lui-même qui avait livré à l'Inquisition romaine de Vienne les pièces à conviction touchant l'affaire de Servet. Et c'était ce même Calvin qui, sept ans auparvant, avait déclaré: 'Si Servet vient à Genève, ... jamais je ne souffrirai qu'il en sorte vivant! ${ }^{\prime}$ Pour comble d'infortune, un concitoyen de Vienne habitant à Genève reconnut et dénonça le savant espagnol lors de son arrivée dans la république protestante, ce qui donna à Calvin l'occasion de réaliser son projet impitoyable. 
Le fait que Calvin avait saisi un voyageur qui n'était que de passage à Genève est pour Voltaire un détail particulièrement scandaleux. 'C'était un attentat inouii, écrit-il dans une lettre de 1768, d'arrêter sans aucun prétexte un sujet de Charles quint qui voyageait sur la foi publique, muni de bons passeports. ${ }^{10} \mathrm{~A}$ ce propos Voltaire note que Servet n'était ni de l'Eglise ni de la ville de Calvin: 'c'était, dit-il, un voyageur tombé dans une caverne de voleurs. ${ }^{11}$

A côté de son travail de médecin et de physiologiste, Servet avait pour passe-temps la spéculation théologique et métaphysique. Plus précisément il tenait à reformuler la doctrine traditionnelle de la Trinité. Ce n'est pas parce qu'il trouve particulièrement intéressantes les idées religieuses de Servet que Voltaire prend la défense de celui-ci. Si, en revanche, le philosophe ne cache pas son admiration pour les recherches qui avaient valu au savant espagnol sa place dans l'histoire de la médecine, il lui reproche d'avoir négligé ce travail utile en faveur de ce qu'il appelle 'des sciences dangereuses. ${ }^{12}$ Il s'agit là, bien entendu, de la théologie. Plus d'une fois dans sa correspondance Voltaire appelle Servet un fou, ${ }^{13}$ bien qu'il adoucisse un peu cette épithète en disant que 'Servet était un fou très honnête. ${ }^{14}$ En même temps Voltaire fait remarquer que la doctrine antitrinitaire pour laquelle Calvin fit brûler Servet n'était pas vraiment nouvelle. Il traduit dans L'Essai sur les moeurs un passage tiré du livre De Trinitatis Erroribus où Servet parle de 'la semence substantielle du Christ,' du 'germe de la génération,' 'des causes séminales et formes archétypes,' etc. Ce genre de discours métaphysique est pour Voltaire exaspérant, pourtant il n'est pas tout à fait original: certains penseurs de l'antiquité chrétienne ${ }^{15}$ et les disciples de Platon tenaient plus ou moins le même langage. Voltaire prétend, sans trop exagérer, que Calvin avait fait exécuter le médecin espagnol 'pour avoir pensé à peu-près comme on pensait dans les trois premiers siècles de l'Eglise.'

Ce n'est donc pas Servet métaphysicien que Voltaire défend, mais plutôt Servet victime du fanatisme. Afin de comprendre pourquoi Voltaire attache tant d'importance à ce martyr du XVIe siècle, nous devons considérer l'idée qu'il se fait du rôle de l'historien. Malgré la passion évidente avec laquelle il écrit, l'auteur de l'Essai sur les moeurs croit viser à l'objectivité dans ses portraits du passé. Dans le chapitre consacré à la mort de Servet, il fait cette déclaration, qui pourrait au premier abord surprendre: 'Nous ne faisons ici que rapporter les faits et les opinions, sans entrer dans aucune controverse, ... et uniquement attaché à la fidélité de l'histoire. ${ }^{16}$ Néanmoins, Voltaire ne se considère pas comme un simple chroniqeur de faits. Il reconnaît que l'historien doit faire des choix, et par conséquent il croit que l'histoire a une certaine fonction didactique. Il incombe à l'historien de créer 'des annales fidèles qui, en 
exposant les crimes, excitent à la vertu. ${ }^{17} \mathrm{Vu}$ dans cette perspective, le crime de Calvin, même rapporté d'une manière que Voltaire croit objective, a la particularité de bien montrer les contradictions intrinsèques de l'intolérance, et ainsi, paradoxalement, d'encourager la liberté de conscience. Plus que d'autres exemples de persécution religieuse, la mise à mort de Servet montre à quel point l'esprit fanatique fonctionne selon sa propre logique, et que cet esprit peut se manifester à l'intérieur de différents systèmes doctrinaux.

Il y a certains thèmes qui reviennent constamment dans les commentaires de cet épisode, commentaires qu'on trouve dans les oeuvres publiées et dans la correspondance de Voltaire. Le philosophe insiste avant tout sur l'hypocrisie de Calvin, et sur l'hypocrisie des pasteurs calvinistes modernes. Si Servet était un fou très honnête, Jean Calvin était, selon les dires de Voltaire dans une de ses lettres, 'le plus malhonnête fanatique qui fut en Europe ... une âme atroce et sanguinaire, un monstre d'orgueil et de cruauté. ${ }^{18}$ A chaque fois qu'il parle de la mort de Servet, Voltaire ne manque pas de noter que Calvin l'avait fait brûler 'à petit feu avec des fagots verts.' Voulant satisfaire son amour-propre par une domination sur les autres, Calvin s'érigea en pape protestant à Genève. ${ }^{19}$ Dans ses rapports avec Servet, le réformateur était poussé par ce sentiment violent que Voltaire appelle 'la haine théologique,' qui est, selon notre auteur, 'la plus implacable de toutes les haines. ${ }^{20}$ Pour illustrer cette idée, Voltaire reproduit dans l'Essai sur les moeurs une lettre que Calvin avait adressée au marquis de Poët huit ans après la mort de Servet. Au sujet de ses ennemis religieux, Calvin y déclare: 'Pareils monstres doivent être étouffés, comme j'ai fait de Michel Servet, Espagnol. ${ }^{21}$ Voltaire publie le texte de cette lettre sans commentaire: Calvin y fait lui-même le portrait de son caractère.

C'est précisément cette haine théologique qui inspira chez Calvin son hypocrisie. Calvin est hypocrite parce qu'il aurait été lui-même brûlé s'il avait mis le pied en France. ${ }^{22}$ Voilà la contradiction fondamentale de toute forme de répression religieuse. Si Calvin et ses disciples demandaient la tolérance en France, à Genève ils usaient de la violence pour imposer leur orthodoxie à eux. ${ }^{23}$ Voltaire n'entend pas, bien sûr, disculper le côté catholique, qui fut tout aussi hypocrite. Cependant, dans ce cas, l'hypocrisie est plutôt une fonction de la raison d'état. En racontant la vie de Calvin dans l'Essai sur les moeurs, Voltaire remarque que François Ier faisait brûler les protestants de France tout en faisant alliance avec ceux d'Allemagne. ${ }^{24}$ Il développe ce thème dans l'article 'Tolérance' du Dictionnaire philosophique, où il évoque l'ironie d'une situation où le très chrétien roi de France réprimait le luthéranisme chez lui alors qu'il s'unissait avec des luthériens et même avec des musulmans contre le catholique Charles Quint. ${ }^{25}$ 
Une autre contradiction sur laquelle Voltaire revient souvent, c'est le fait que la théologie calviniste a évolué au point où plusieurs pasteurs calvinistes soutiennent maintenant des idées semblables à celles qui avaient provoqué l'exécution de Servet. En 1757 d'Alembert, dans l'article sur Genève qu'il prépara pour l'Encyclopédie, affirme qu'un certain nombre de pasteurs genevois ' $n$ ' ont $d$ 'autre religion qu' un socinianisme parfait,' c'est-à-dire, qu'ils n'acceptent plus la doctrine orthodoxe de la Trinité. Ils rejettent, d'après l'article de d'Alembert, 'tout ce qu' on appelle mystères, ... s'imaginant que le premier principe d'une religion véritable, est de rien proposer à croire qui heurte à la raison.' Ecrivant à d'Alembert quatre ans plus tard, Voltaire appelle ces pasteurs des 'prédicants ... servétiens. ${ }^{26}$ Ailleurs il parle d'une 'assez jolie révolution dans l'histoire del'esprit humain' où les pasteurs calvinistes, qui avaient autrefois rejeté la doctrine catholique du purgatoire, rejettent aujourd'hui l'idée des peines éternelles de l'enfer. En fait ils n'ont fait que transformer l'enfer en purgatoire. Autrement dit, les calvinistes contemporains sont bien des hommes du siècle des lumières. 'Je vois de mes fenêtres, écrit Voltaire de sa propriété les Délices en 1759, la ville où régnait Jean Chauvin le Picard, ditCalvin, et la placeoù il fit brûler Servet pour lebien de sonâme. Presque tous les prêtres de ce pays-ci pensent aujourd'hui comme Servet, et vont même plus loin que lui ... Admirez, je vous prie, les contradictions de ce monde: voilà des gensqui sont presque ouvertementsectateurs deServet, et qui m'injurient pour avoir trouvé mauvais que Calvin l'ait fait brûler à petit feu avec des fagots verts! ${ }^{27}$

Après la publication de l'article de d'Alembert, les pasteurs de Genève avaient publié une déclaration dans laquelle ils essayaient de repousser les allégations de socinianisme. Cependant, Voltaire n'a jamais admis que lui et d'Alembert eussent tort, et un historien moderne qui a étudié la question conclut qu'au moins cinq membres du clergé genevois soutenaient des thèses peu orthodoxes sur la Trinité. ${ }^{28}$

Pour Voltaire, l'hypocrisie qui caractérise l'attitude de Calvin et de ses successeurs n'est pas fonction de la théologie calvinienne, ni même de la psychologie égoïste des personnes en question. Elleest plutôt un élément constituant du virus du fanatisme, qui peut infecter n'importe quel système théologique. Par conséquent il aurait été tout à fait possible que Servet, s'il s'était trouvé à la tête de la Réforme genevoise, persécute Calvin et d'autres protestants qui n'acceptaient pas sa théologie antitrinitaire. Dans le Poème surlaloi naturelle (1752), Voltaireécrit les vers suivạnts:

Servet fut en personne immolé par Calvin.

Si Servet en Genève eût été souverain,

Il eût, pour argument contre ses adversaires,

Fait serrer d'un lacet le cou des trinitaires. ${ }^{29}$ 
En somme, les contradictions qui se rattachent au cas de Servet montrent clairement l'illogisme de la pratique de l'intolérance. Elles peuvent se réduire à ce principe: si vous avez le droit de me tuer parce que je ne suis pas d'accord avec vous, j'ai le droit de vous tuer pour la même raison.

Voltaire admet qu'il y a plus d'un type de fanatisme. Au fanatisme pacifique de Saint François d'Assise ou des fakirs de l'Inde, par exemple, il oppose dans une lettre de 1766 'celui des juges qui firent brûler le conseiller Dubourg et le médecin Servet. ${ }^{30}$ Ce genre de fanatisme malveillant fonctionne selon sa propre logique pervertie, qui arrive même à séparer les actes d'intolérance des intérêts particuliers des persécuteurs. 'Cette dureté de Calvin, admet Voltaire, était jointe au plus grand désintéressement. ${ }^{31}$ La logique du fanatisme contredit aussi les principes de la loi naturelle et de la justice humaine. Deux ans après la publication de l'Essai sur les moeurs, son auteur condamne les juges de Servet en déclarant qu'ils étaient 'des cannibales en manteau noir' qui 'violèrent ouvertement tous les droits des nations. ${ }^{, 32}$ La mort de Servet fut 'un assassinat juridique,' ou encore 'un véritable assassinat commis en cérémonie,' 'une violation criminelle du droit des gens. ${ }^{33} \mathrm{Qu}$ 'une telle atteinte à la justice humaine ait pu se produire dans une république constitue une leçon en sciences politiques. 'Si vous songez, affirme Voltaire, que le républicain Jean Calvin ... fit brûler tout vif et avec des fagots verts un Espagnol qui s'exprimait sur la trinité autrement que lui, en vérité, monsieur, vous conclurez qu'il n'y a pas plus de vertu dans les républiques que dans les monarchies. ${ }^{, 34}$ Voilà une autre contradiction: on s'attendrait à ce qu' une république soit moins autoritaire qu' une monarchie, mais l'exécution de Servet prouve que ce n'est pas le cas. De même, la doctrine protestante, qui en apparence est plus respectueuse des droits de l'individu que le catholicisme, ne l'est pas en réalité. 'Sa religion, écrit Voltaire dans l'Essai sur les moeurs, est conforme à l'esprit républicain, et cependant Calvin avait l'esprit tyrannique. ${ }^{35}$

Voltaire se passionne pour le cas de Servet parce que pour lui l'histoire est encore vivante; ce cas est important parce que l'esprit qui mena Servet au bûcher est le même esprit qui maintenant incite les persécuteurs des Calas et des Sirven. D'ailleurs, en insistant sur l'intolérance du fondateur du protestantisme français, le patriarche de Ferney peut poursuivre sa campagne en faveur de la liberté religieuse sans s'exposer à des accusations de parti-pris contre l'Eglise catholique. Lorsqu'il prend la défense d'un personnage historique qui avait été mis à mort à cause de ses idées particulières et excentriques, Voltaire peut montrer que les individus et non seulement les groupes sociaux ont droit à la liberté de pensée. A une époque où la question de la tolérance se posait surtout en termes de droits collectifs, la mise en valeur des droits 
individuels par Voltaire l'historien marque une étape importante dans l'évolution des droits de la personne.

\section{JAMES FORSYTHE MACLEAN \\ Memorial University}

\section{Notes}

1 Dictionnaire philosophique, texte établi par Raymond Naves (Paris: Garnier, 1954), p. 173. L'article 'Dogmes' fut publié en 1765.

2 Voir surtout Chant II, vers 176-358, dans l'édition critique par O.R. Taylor, Voltaire 2, pp. 398-409. L'indication 'Voltaire' suivie d'un chiffre arabe désigne les Oeuvres complètes de Voltaire/Complete Works of Voltaire, éd. Th. Besterman et al. (Genève, Banbury, Oxford, 1968ss.). L'indication 'M.' suivie d'un chiffre romain et d'un chiffre arabe renvoie au tome et à la page de l'édition Garnier des Oeuvres complètes de Voltaire, éd. L. Moland (Paris, 1877-1885).

3 Theodore Bestermann, Voltaire, troisième édition (Oxford: Basil Blackwell, 1976) p. 103.

4 Voltaire à Charles Jean François Hénault, le 26 février 1768, dans Best.D14779, Voltaire 117, p. 135. L'indication 'Best. $D$ ' suivie d'un nombre désigne le numéro de la lettre dans la Correspondence and Related Documents. The Complete Works of Voltaire, t. 85-135, éd. Th. Bestermann (Genève, Banbury, Oxford, 1968-77). Nous conservons l'orthographe de Voltaire.

5 Voltaire à Charles Jean François Hénault, le 14 mars 1768, Best.D14832, Voltaire 117, pp. 180-181.

6 Commentaire historique, M.i.98.

7 Le 6 mars 1759, Best.D8158, Voltaire 104, p. 19. Nicolas Antoine (1602-1632) fut un pasteur genevois exécuté dans la ville pour ses tendances judaïsantes.

8 R. Bainton, The Travail of Religious Liberty (Hamden, Conn.: Archon, 1971), p. 83s.

9 Lettre de Calvin à G. Farel, le 13 février 1546, citée dans F. Buisson, Sébastien Castellion (Paris: Hachette, 1892), t. I, p. 336.

10 Voltaire à Charles Jean François Hénault, le 26 février 1768, in Best.D14779, Voltaire 117, p. 135.

11 Voltaire à Charles Jean François Hénault, Best.D14832, Voltaire 117, p. 181 .

12 Essai sur les moeurs, M.xii.306.

13 Par exemple Best.D7913 (Voltaire à Charles de Brosses), Voltaire 103, p. 221.

14 Voltaire à Charles Jean François Hénault, Best.D14832, Voltaire 117, p. 180.

15 Il nomme Origène.

16 Essai sur les moeurs, M.xii.308.

17 J.H. Brumfitt, Voltaire Historian, (Oxford: University Press, 1958), p. 96. 
18 Voltaire à Charles Jean François Hénault, le 14 mars 1768, Best.D14832, Voltaire 117, 1 p. 180-181. Cf. Voltaire à Jean Vernes, le 12 janvier 1758, Best.D7580,

Voltaire 102, p. 365.

19 Essai sur les moeurs, M.xii.304.

20 Ibid., pp. 306-307.

21 Ibid., p. 308.

22 Ibid., p. 307; Voltaire à Charles Jean François Hénault, Best.D14779, Voltaire 117, p. 135.

23 Essai sur les moeurs, M.xxii.307.

24 Ibid., p. 305.

25 Dictionnaire philosophique, p. 403.

26 Le 6 janvier 1761, Best.D9523, Voltaire 106, p. 443.

27 Mémoires (1759), M.i.54.

28 Graham Gargett, Voltaire and Protestantism (Oxford: The Voltaire Foundation, 1980), p. 142s.

29 M.ix.453.

30 Voltaire à Jacob Vernes, le 4 septembre 1766, Best.D13651, Voltaire 115, p. 68. Anne du Bourg (1521-1559), conseiller clerc au parlement de Paris, fut exécuté pour son calvinisme.

31 Essai sur les moeurs, M.xii.309.

32 Voltaire à Charles Jean François Hénault, le 14 mars 1768, Best.D14832, Voltaire 117, pp. 180-181.

33 Voltaire à Charles Jean François Hénault, le 26 février 1768, Best.D14779, Voltaire 117, p. 135.

34 Voltaire au chevalier de R...x, le 20 septembre 1760, Best.D9247, Voltaire 106, p. 136.

35 M.xii.305. 